\title{
Study of Incremental Updating Algorithm for Association Rules
}

\author{
An Hongmei, Chen Ping \\ Technology Department Maanshan Teachers' School \\ Maanshan, 243000, China
}

\author{
Huang Lijing \\ School of Information Science \& Engineering \\ Hebei University of Science and Technology \\ Shijiazhuang, 050018, China
}

\begin{abstract}
Discover frequent itemsets is the key problem of mining association rules, and the expenditure of this process is high. So, the research of incremental updating algorithms is particular important. This paper presents a comprehensive study on the performance of incremental updating algorithms that currently available, such as IUA [1] which makes full use of frequent itemsets already got. Based on the shortcomings of IUA, presents a new algorithm AIUA as an improved algorithm of IUA.
\end{abstract}

Keywords-Data mining, Association rules, Incremental updating

\section{INTRODUCTION}

Data mining, also known as knowledge discovery from database ( KDD ) [2], is an intelligent data analysis technique in the 1980's then gradually developed. It is a procedure to obtain valid, new, potentially useful and ultimately comprehensible information and knowledge from a large number of data that stored in a database, data warehouse or other repository.

As one of the many ways that data mining uses, association rules mining is first proposed by Agrawal in 1993. Its purpose is to find valuable knowledge that describes the affiliation between data items from a large number of data and its successful application in business and so on makes it the most mature, important, active research of data mining. But, a lot of work has focused on mining algorithms until the end of last century, and the database that association rules used is extremely large, so not only designing of efficient algorithms for mining association rules but also the update, maintenance and management of the association rules which have been found are both important. Thus, since then many scholars have done a lot in the update of association rules.

This paper considered the update when the minimum_support(min_sup) and minimum confidence(min conf) changed, pointed out the shortcomings of IUA, and upon the shortcomings of IUA, presented AIUA. AIUA improves the efficiency of mining in ensuring the validity.

\section{AsSOCiATION RULES Mining}

Formal description of association rules is as follows:

Let $I=\left\{i_{1}, i_{2}, \ldots, i_{m}\right\}$, this is a data items set, $D$ is a data set that associated with task, namely a transaction database, each transaction $\mathrm{T}$ is a subset of data items set, namely
$\mathrm{T} \subseteq \mathrm{I}$; each transaction contains an identification code TID. Let $\mathrm{A}$ is a data items set, if and only if $\mathrm{A} \subseteq \mathrm{T}$, we said transaction $\mathrm{T}$ contains $\mathrm{A}$. An association rule is an implication that in the form of " $\mathrm{A} \Rightarrow \mathrm{B}$ ", where $\mathrm{A} \subset \mathrm{I}, \mathrm{B} \subset \mathrm{I}$ and $\mathrm{A} \cap \mathrm{B}=\Phi$. The rule $\mathrm{A} \Rightarrow \mathrm{B}$ is true in the transaction database $\mathrm{D}$, and with s support and c confidence, means that in the transaction database $\mathrm{D}$ has $\mathrm{s}$ ratio of the transaction $\mathrm{T}$ contains $\mathrm{A} \cup \mathrm{B}$ data items, and has $\mathrm{c}$ ratio of the transaction T to meet "if contains A then contains B". Concrete description is as follows:

$$
\begin{aligned}
& \operatorname{support}(A \Rightarrow B)=P(A \cup B) \\
& \text { confidence }(A \Rightarrow B)=P(B \mid A)
\end{aligned}
$$

Rules that meet the given min_sup and min_conf are called strong association rules. Mining association rules is to find out all strong rules from transaction database.

The two steps of mining association rules are as follows:

Step 1: Screen out all itemsets (nonempty subset of data itemsets I) with user-specified min sup from transaction database D. The itemsets with the min_sup are called frequent itemsets, otherwise known as non-frequent itemsets.

Step 2: Using frequent itemsets to generate all association rules. For each frequent itemset $\mathrm{A}$, find out all nonempty subset of $\mathrm{A}$ as $\mathrm{a}$, if the ratio of support (A) / support (a) $>$ min_conf, then generate association rule $\mathrm{a} \Rightarrow(\mathrm{A}-\mathrm{a})$,in which min_conf is the user-specified minimum confidence.

\section{INCREMENTAL UPDATING ALGORITHM FoR ASSOCIATION RULES}

The problem of updating association rules can be divided into two categories: first, given a transaction database D, keepping the min sup unchanged, when a new transaction database $d$ added to D, such as literature [3], [4], [5] and [6]; second, the transaction database $\mathrm{D}$ remains unchanged, while the min_sup and min_con changed, such as literature [1]. At present, there are many mature algorithms available, these algorithms take advantage of all the original information to efficiently find out all the new association rules or frequent itemsets, and effectively solve the above problems.

In the process of mining association rules, in order to discover the unknown and of potentially useful association rules in advance, users need to constantly adjust the min sup and min_conf to gradually focus to those association rules that they really interested, this will be a dynamic, interactive 
process. Therefore, we urgent need an efficient updating algorithm to meet user's demand of faster response time.

This paper and literature [1] consider the second. Due to limited space, this paper is no longer pay attention to the detailed description of IUA, please see [1], and the symbols used in this paper are in the same meaning except where extremely noted.

\section{A. The shortcomings of IUA}

In the join phase of generating candidate itemsets, IUA presents a new candidate k-itemsets generation function named iua-gen $\left(\mathrm{L}^{j}\right)$ to generate $\mathrm{C}^{k}$

Throw careful study you will find that some large itemsets will be deleted by mistake, because the subsets of k1 length that included in $\mathrm{L}_{k}^{3}$ must be large itemsets, but not necessarily include in $\mathrm{L}_{k-1}^{3}$, they may also included in $\mathrm{L}_{k-1}^{1}$, or $\mathrm{L}_{k-1}^{2}$. For example: a case in point is that in $\mathrm{L}_{k}^{3}$, only one item is included in $\mathrm{L}_{1}$, the remaining $\mathrm{k}-1$ items are included in $\mathrm{L}_{1}$, its $\mathrm{k}-1$ subsets include one kind that all the items are included in $\mathrm{L}_{1}$, means that they are included in $\mathrm{L}_{k-1}^{2}$. Similarly, there is also one kind that all the items are included in $\mathrm{L}_{1}$. To solve this problem, this paper presents improved algorithm AIUA.

B. AIUA

For $\mathrm{C}^{k}$ and $\mathrm{C}^{2}$, from the beginning loop $\mathrm{k}=2$, that is $\mathrm{k}$ $\geq 2$, we directly use function apriori gen of Apriori, $\mathrm{C}^{k}$ generated by self-join of $\mathrm{L}^{k-1} ; \mathrm{C}^{k}$ generated by self-join of

$$
\begin{array}{rl}
\mathrm{L}^{k-1} & \\
1 & 1 \\
\mathrm{C}^{k} & =\text { apriori_gen }\left(\begin{array}{c}
\mathrm{L}^{k-1} \\
2
\end{array}\right)-\mathrm{L}_{\mathrm{k}} ; \\
2 & \text { Capriori_gen }\left(\mathrm{L}^{k-1}\right) ; \\
3
\end{array}
$$

Before generating $\mathrm{C}^{k}$, we divide it into three categories: $31 \quad 32 \quad 33 \quad 31$ $\mathrm{C}^{k}, \mathrm{C}^{k}$ and $\mathrm{C}^{k} \cdot \mathrm{C}^{k}$ contains all k-itemsets that consists of one item included in $\mathrm{L}_{1}$ and $\mathrm{k}-1$ items included in $\mathrm{L}_{1}$; $\mathrm{C}^{33}$ contains all k-itemsets that consists of $\mathrm{k}-1$ items included in $\mathrm{L}_{1}$ and one item included in $\mathrm{L}_{1}$; $\mathrm{C}^{k}$ contains all other candidate k-itemsets. According to the definition above we know that $\mathrm{C}_{k}^{31}$ generated by connection of $\mathrm{L}_{k-1}^{2}$ and $\mathrm{L}_{k-1}^{31}$; $\mathrm{C}_{k}^{33}$ generated by connection of $\mathrm{L}_{k-1}^{1}$ and $\mathrm{L}_{k-1}^{33} ; \mathrm{C}_{k}^{32}$ generated by self-connection of $\mathrm{L}_{k-1}^{3}$. Accordingly $\mathrm{L}^{k}$ is also divided into three categories, $\mathrm{L}^{k 1}, \mathrm{~L}^{k}$ and $\mathrm{L}^{k 3}$.
For generation of $\mathrm{C}_{k}^{3}$, when $\mathrm{k}=2$ and $\mathrm{k}=3$, it does not have the classification as described above, so raise them alone, the beginning loop from $\mathrm{k}=4$.

(1) $\mathrm{k}=2 \mathrm{C}^{2}$ generated by join of $\mathrm{L} 1$ and $\mathrm{L}^{1}$, via function aiua_gen $2\left(\mathrm{~L}_{1}, \mathrm{~L} 1\right)$ :

(1)join:

$$
\begin{aligned}
& \text { insert into } \mathrm{C}^{2} \\
& \text { select p.item1, q.item1 } \\
& \text { from L1p, } \mathrm{L}^{1} \mathrm{q} \\
& \text { (2)pruning: }
\end{aligned}
$$

for all itemsets $\mathrm{c} \in \mathrm{C}^{2}$ do for all 1-items of $\mathrm{c}$ do

$$
\begin{aligned}
& \text { if }\left(s \notin L^{1}\right) \text { then } \\
& \text { delete } c \text { from } \mathrm{C}^{2} \text {; }
\end{aligned}
$$

(2) $\mathrm{k}=3$ divides $\mathrm{C}^{3}$ into two categories: $\mathrm{C}^{31}-$ generated by join of $\mathrm{L}^{2}$ and $\mathrm{L}_{1}^{3}$ (get $\mathrm{L}_{3}^{31}$ by pruning), $\mathrm{C}^{33}-$ generated by join of $\mathrm{L}^{2}$ and $\mathrm{L}^{2}$ (get $\mathrm{L}^{3}$ by pruning), via function aiua_gen $3\left(\mathrm{~L}^{2}, \mathrm{~L}^{2}, \mathrm{~L}^{2}\right)$ :

(1)join:

insert into $\mathrm{C}^{3}$

select p.item1,p.item2,q.item2

from $\mathrm{L}^{2} \mathrm{p}, \mathrm{L}^{2} \mathrm{q}$

where $\mathrm{p} . \mathrm{item} 1=\mathrm{q}$. item 1

insert into $\mathrm{C}^{3}$

select p.item1,p.item2,q.item2

from $L^{\frac{1}{2}} \mathrm{p}, \mathrm{L}^{2} \mathrm{q}$

where p.item $1=$ q.item 1

(2)pruning:

for all itemsets $\mathrm{c} \in \mathrm{C}^{3}$ do for all 2-items of $\mathrm{c}$ do

$$
\begin{aligned}
& \text { if }\left(\mathrm{s} \notin \mathrm{L}^{2}\right) \text { then } \\
& \text { delete } \mathrm{c} \text { from } \mathrm{C}^{3} \text {; }
\end{aligned}
$$

(3) $\mathrm{k}=4$ loop start

This paper presents the improved function aiua_gen $\left(\mathrm{L}^{k-1}\right.$, $\left.\mathrm{L}^{2-1}, \mathrm{~L}^{k-1}, \mathrm{~L}^{k-1}, \mathrm{~L}^{k-1}, \mathrm{~L}^{k-1}\right)$ of iua_gen $\left(\mathrm{L}^{j}\right)$, including the following two steps:

(1)join:

$$
\text { insert into } \mathrm{C}^{31}
$$


select p. item1, p. item2, .., p. item(k-2), p. item(k-1), q. item $(\mathrm{k}-1)$

from $\mathrm{L}^{2-1} \mathrm{p}, \mathrm{L}^{k-1} \mathrm{q}$ where $\mathrm{p}$. item $1=\mathrm{q}$. item $1, \mathrm{p}$. item $2=$ q. item $2, \ldots$, p.item $(\mathrm{k}-2)=\mathrm{q}$. item $(\mathrm{k}-2)$

insert into $\mathrm{C}^{k}$

select p. item1, p. item $2, \ldots$, p. item(k-2), p. item(k-1), q. item(k-1)

from $\mathrm{L}^{1}{ }^{k-1} \mathrm{p}, \mathrm{L}^{k-1} \mathrm{q}$ where $\mathrm{p}$. item $1=\mathrm{q}$. item $1, \mathrm{p}$. item $2=$ q. item $2, \ldots$, p.item $(\mathrm{k}-2)=$ q.item $(\mathrm{k}-2)$

insert into $\mathrm{C}^{k}$

select p. item1, p. item $2, \ldots$, p. item(k-2), p. item(k-1), q item(k-1)

from $\mathrm{L}^{3-1} \mathrm{p}, \mathrm{L}^{k-1} \mathrm{q}$ where $\mathrm{p}$. item $1=\mathrm{q}$. item $1, \mathrm{p}$. item $2=$ q. item $2, \ldots$, p.item $(\mathrm{k}-2)=$ q.item $(\mathrm{k}-2)$

(2)pruning:

for all itemsets $\mathrm{c} \in \mathrm{C}^{k}$ do

for all (k-1) -subsets s of $\mathrm{c}$ do

$$
\begin{aligned}
& \text { if }\left(\mathrm{s} \notin \mathrm{L}^{k-1}\right) \text { then } \\
& \text { delete } \mathrm{c} \text { from } \mathrm{C}^{k} \text {; }
\end{aligned}
$$

The correctness of AIUA can be proved by the previous definition and discussion, its analysis process is the same as the proof of Apriori, no further explanation.

Description of AIUA:

Input:

(1)transaction database $\mathrm{D}$;

(2)old support s, new support s';

(3)candidate itemsets $\mathrm{L} \mathrm{k}$ with $\mathrm{s}$.

Output: candidate itemsets with s'.

Begin:

1) $\mathrm{L}^{1}=\{$ new frequent 1 -itemsets $\} ; \mathrm{L}^{1}=\mathrm{L}^{1} \cup \mathrm{L} 1$;

2) for $\left(\mathrm{k}=2 ; \mathrm{L}^{k-1} \neq \Phi ; \mathrm{k}++\right)$ do begin

3) $\mathrm{C}^{k}=$ apriori_gen $\left(\mathrm{L}_{2}^{k-1}\right)-\mathrm{Lk}$;

4) $\mathrm{C}^{k}=$ apriori_gen $\left(\mathrm{L}^{k-1}\right)$;

5) $\mathrm{C}^{3}=\Phi$;

6) if $(\mathrm{k}==2)$ then

7) $\mathrm{C}^{k}=$ aiua_gen2 $\left(\mathrm{L} 1, \mathrm{~L}^{1}\right)$;

8) else if $(k==3)$ then

$\begin{array}{llll}3 & 1 & 2 & 3 \\ \mathrm{C}^{k} & & \mathrm{C}^{2} & \end{array}$

9) $\mathrm{C}^{k}=$ aiua $\_$gen $3\left(\mathrm{~L}^{2}, \mathrm{~L}^{2}, \mathrm{~L}^{2}\right)$;

10) else ${ }_{32}{ }^{3} k=$ aiua_ gen $\left(\mathrm{L}^{1} k-1\right.$, $\left.\mathrm{L}^{k-1}, \mathrm{~L}^{k-1}, \mathrm{~L}^{k-1}, \mathrm{~L}^{k-1}, \mathrm{~L}^{k-1}\right)$;

11) for all transactions $t \in D$ do begin

12) $\quad \mathrm{Ct} 1=\operatorname{subset}\left(\mathrm{C}^{k}, \mathrm{t}\right)$;
13) for all candidates $\mathrm{c} \in \mathrm{Ct} 1$ do

14) c.count++;

15) $\mathrm{Ct} 2=\operatorname{subset}\left(\mathrm{C}^{k}, \mathrm{t}\right)$;

16) for all candidates $\mathrm{c} \in \mathrm{Ct} 2$ do

17) c.count ++ ;

18) $\mathrm{Ct} 3=\operatorname{subset}\left(\mathrm{C}^{k}, \mathrm{t}\right)$;

19) for all candidates $\mathrm{c} \in \mathrm{Ct} 3$ do

20) c.count++;

21) end

22) $\mathrm{L}^{k}=\left\{\mathrm{c} \in \mathrm{C}^{k} \mid \mathrm{c} . \operatorname{count} \geqslant \mathrm{s}^{\prime}\right\} ; \mathrm{L}^{k}=\mathrm{L}^{k} \cup \mathrm{Lk}$;

$$
2 \quad 2
$$

23) $\mathrm{L}^{k}=\left\{\mathrm{c} \in \mathrm{C}^{k} \mid \mathrm{c} . \mathrm{count} \geqslant \mathrm{s}^{\prime}\right\}$;

$$
3 \quad 3
$$

24) $\mathrm{L}^{k}=\left\{\mathrm{c} \in \mathrm{C}^{k} \mid \mathrm{c} \cdot \mathrm{count} \geqslant \mathrm{s}^{\prime}\right\}$;

25) $\mathrm{L}^{k}=\mathrm{L}^{k} \cup \mathrm{L}^{k} \cup \mathrm{L}^{k}$

26) end

27) Answer $=\cup_{k} L^{k}$

\section{PERFORMANCE ANALYSIS}

\section{A. Compare to Apriori}

The simplest and intuitionistic approach for updating association rules is to run Apriori once again in the original database with new support, the following will compare AIUA to rerunning Apriori once again. Apriori first scans the database once to count all the candidate 1-itemsets, but all of the frequent 1-itemsets with the original support are removed from AIUA, and the amount of itemsets that included in $\mathrm{L}_{1}$ is large, so this will greatly reduce the number of itemsets which need to calculate the support. In addition, during the subsequent course of scanning the database, divide $\mathrm{C}_{\mathrm{k}}$ into three categories, and remove $\mathrm{L}_{\mathrm{k}}$ from $\mathrm{C}_{k}^{1}$, these also reduce the number of candidate items. Reducing the number of candidates can save computing time and storage space that required.

\section{B. Compare to IUA}

Although IUA reduced the generation of candidate itemsets to a great extent, but when it generates $\mathrm{C}^{3}$, join a frequent $\mathrm{j}$-itemset $(1 \leq \mathrm{j} \leq \mathrm{k}-1)$ of $(1)$ to a frequent $(\mathrm{k}-\mathrm{j})$ itemset of (2), this also generates a large number of useless candidates, then AIUA does well, it not only reduces the candidate itemsets, but also makes connections only when the conditions meet the connection condition, so it greatly reduces the number of itemsets that need test. The efficiency will be greatly improved when the number of frequent $\mathrm{k}$ itemsets is large.

In addition, in the case that the change of min_sup is smaller, the number of $L_{1}$ is small than the number of $L_{1}$, so the efficiency of AIUA will be more significant than the original algorithm. 


\section{SUMMARIES}

This paper studyed the second type of association rules updating problem, presented an improved efficient algorithm

AIUA. Moreover, since AIUA divided $\mathrm{C}^{k}$ into three categories, multiprocessor can achieve parallel computing. This is very useful to reduce the running time of large-scale program. But AIUA also has its own drawbacks, such as explicitness decreases.

In this paper, just considered the min_sup changes of the association rules updating problem, how to implement the update when the transaction database changes (including the amount of data increases and decreases) will be the future work.

\section{ACKNOWLEDGEMENT}

This work is supported by the 2011 Anhui Provincial Natural Science Research Project of University named
"Study of Contract Programming Development Environment Based on AOP" under contract number KJ2011B171.

\section{REFERENCES}

[1] FENG Yu-cai; FENG Jian-lin. Incremental Updating Algorithms for Mining Association Rules. Journal Software, 1998,9(4), PP:301-306

[2] Tan Pang-Ning; Steinbach.M.; Kumar.V. Introduction to Data Mining. China Machine Press. 2010

[3] CAI Jin; XUE Yong-Sheng; LIN Li; ZHANG Dong-Zhan. Updating Algorithm for Association Rules Based on Fully Mining Incremental Transactions. Computer Science. Vol.34 No.2 2007, PP:220-233

[4] TIE Zhi-xin; YU Rui-zhao. An Efficient Algorithm for Incremental Updating Association Rules. ournal of Zhejiang Sci-Tech University. Vol.25 No.2 2008(3), PP:169-173

[5] ZHENG Wen-zheng. Research on Increment Association Rules Mining Algorithm. Computer and Modernization, 2009(2), PP:92-97

[6] ZHANG Yong-tao; ZHANG Gang-hua. Improved Updating Algorithm Assciation Rules. Computer and Information Technology, 2010(1), PP:4-7 